\title{
BMJ Open Prenatal exposure to cannabis and maternal and child health outcomes: a systematic review and meta-analysis
}

\author{
J K L Gunn, ${ }^{1}$ C B Rosales, ${ }^{2}$ K E Center, ${ }^{3}$ A Nuñez, ${ }^{4}$ S J Gibson, ${ }^{5}$ C Christ, ${ }^{6}$ \\ JE Ehiri ${ }^{5}$
}

To cite: Gunn JKL,

Rosales CB, Center KE, et al.

Prenatal exposure to

cannabis and maternal and

child health outcomes:

a systematic review and

meta-analysis. BMJ Open

2016:6:e009986.

doi:10.1136/bmjopen-2015009986

- Prepublication history and additional material is available. To view please visit the journal (http://dx.doi.org/ 10.1136/bmjopen-2015009986)

Received 14 September 2015 Revised 4 January 2016 Accepted 9 February 2016

CrossMark

For numbered affiliations see end of article.

Correspondence to Professor John Ehiri; jehiri@email.arizona.edu

\section{ABSTRACT}

Objective: To assess the effects of use of cannabis during pregnancy on maternal and fetal outcomes.

Data sources: 7 electronic databases were searched from inception to 1 April 2014. Studies that investigated the effects of use of cannabis during pregnancy on maternal and fetal outcomes were included.

Study selection: Case-control studies, cross-sectional and cohort studies were included.

Data extraction and synthesis: Data synthesis was undertaken via systematic review and meta-analysis of available evidence. All review stages were conducted independently by 2 reviewers.

Main outcomes and measures: Maternal, fetal and neonatal outcomes up to 6 weeks postpartum after exposure to cannabis. Meta-analyses were conducted on variables that had 3 or more studies that measured an outcome in a consistent manner. Outcomes for which meta-analyses were conducted included: anaemia, birth weight, low birth weight, neonatal length, placement in the neonatal intensive care unit, gestational age, head circumference and preterm birth.

Results: 24 studies were included in the review. Results of the meta-analysis demonstrated that women who used cannabis during pregnancy had an increase in the odds of anaemia (pooled OR ( $p O R$ ) $=1.36: 95 \% \mathrm{Cl} 1.10$ to 1.69) compared with women who did not use cannabis during pregnancy. Infants exposed to cannabis in utero had a decrease in birth weight (low birth weight pOR $=1.77: 95 \% \mathrm{Cl} 1.04$ to 3.01 ; pooled mean difference (pMD) for birth weight=109.42 g: 38.72 to 180.12) compared with infants whose mothers did not use cannabis during pregnancy. Infants exposed to cannabis in utero were also more likely to need placement in the neonatal intensive care unit compared with infants whose mothers did not use cannabis during pregnancy ( $\mathrm{pOR}=2.02: 1.27$ to 3.21 ).

Conclusions and relevance: Use of cannabis during pregnancy may increase adverse outcomes for women and their neonates. As use of cannabis gains social acceptance, pregnant women and their medical providers could benefit from health education on potential adverse effects of use of cannabis during pregnancy.

\section{INTRODUCTION}

Global trends in drug use indicate that cannabis remains the drug of choice in developed and developing countries, with

\section{Strengths and limitations of this study}

- Anaemia was the most widely discussed maternal outcome in the cannabis-pregnancy literature. Women who used cannabis during pregnancy may have an increase in the odds of anaemia compared with women who did not use cannabis during pregnancy.

- Infants exposed to cannabis in utero had decreased birth weight and were more likely to need placement in the neonatal intensive care unit or intensive care unit compared with infants whose mothers did not use cannabis during pregnancy.

- Many cannabis users are often tobacco or alcohol users; hence, determining a cannabisonly effect (excluding the presence of tobacco and alcohol) was currently not possible, as most studies did not exclude participants with polysubstance use. Future research in the area of cannabis and maternal and fetal health needs to exclude polysubstance use.

- Very few outcomes were measured using the same cut-offs across multiple articles; therefore, few variables included here could be clearly interpreted. Additionally, many studies reported unique maternal and fetal outcomes not reported in other studies; therefore, definitive conclusions could not be drawn.

- Reliance on self-reported measures of use of cannabis, may have underestimated the prevalence of drug use during pregnancy due to social desirability.

$2.5-4.9 \%$ of the world's population aged 15-64 years using cannabis. ${ }^{1}{ }^{2}$ Regional trends in use of cannabis indicate notably high rates of use in West and Central Africa (12.4\%), North America (12.1\%), Colombia $(15.2 \%)$ and Oceania $(10.8 \%) .^{2}$

Historically, cannabis was of little concern to public health officials. However, recent reports indicate an increase in adverse health outcomes linked to regular use of cannabis that include: motor vehicle accidents, drug-induced psychotic symptoms and psychotic disorders, HIV, hepatitis B and C, 
infective endocarditis, and tuberculosis. ${ }^{3}$ Also, between 2003 and 2012, an increase in the proportion of cannabis-related admissions to substance abuse treatment services was demonstrated in Western and Central Europe (from $19 \%$ to $25 \%$ ), Eastern and South-Eastern Europe (from $8 \%$ to $15 \%$ ), Latin America and the Caribbean (from 24\% to 40\%), and Oceania (from $30 \%$ to $46 \%) .{ }^{2}$ In Africa, cannabis contributed $60 \%$ of the proportion of treatment admissions due to illicit drugs. ${ }^{4}$ With recent reports indicating widespread use of cannabis, questions have been raised about the extent to which cannabis may affect maternal health and fetal development.

There is a paucity of well-designed studies that assess the effects of prenatal exposure to cannabis on maternal and fetal health outcomes. ${ }^{5}$ More importantly, the results of the few available studies are often conflicting, and conclusions are complicated because studies often include participants with polysubstance use. The effects of in utero exposure to other illicit drugs, such as cocaine, have been widely studied; however, less is known about the effects of cannabis on fetal growth and development, or its effects on pregnant women. This paper summarises, and critically appraises, existing literature on the effects of prenatal exposure to cannabis on women and their neonates. The data summarised here may be useful in guiding policy, practice and future research on the benefits and harms associated with use of cannabis during pregnancy. As use of cannabis gains social acceptance, pregnant women and their medical providers could benefit from health education on potential adverse effects of use of cannabis during pregnancy.

\section{METHODS}

\section{Search strategy}

We searched the following databases from inception to 1 April 2014: PubMed/MEDLINE, Ovid/MEDLINE, CINAHL/EBSCO, PsychInfo/EBSCO, Web of Science, Sociological Abstracts and EMBASE. A broad range of keywords that focused on cannabis and maternal, fetal, perinatal and neonatal outcomes were used to search each database (see online supplement 1 ). These keywords were first established in PubMed and then formatted to each individual database in an attempt to find a range of articles from a variety of different fields. In addition, all references and review articles were hand searched for other potentially relevant articles. Finally, in order to ascertain if there were any applicable unpublished or ongoing details of the studies assessed, we attempted to contact the lead author of each study included in this manuscript.

\section{Inclusion/exclusion criteria}

\section{Type of studies}

As previously stated in the study protocol, ${ }^{6}$ randomised controlled trials, case-control, cross-sectional and cohort studies that investigated the effects of prenatal use of cannabis on maternal, fetal, perinatal and neonatal outcomes were eligible for inclusion in this systematic review. Studies must have included data on women who used cannabis during pregnancy. In order to rule out effects of other illicit drugs (eg, cocaine, methadone), only studies that reported outcomes of prenatal use of cannabis while excluding other illicit substances were included in this study. Owing to low numbers of studies that account for concurrent cannabis, alcohol and tobacco use, studies reporting outcomes of in utero exposure to cannabis in the presence of smoking tobacco and consuming alcohol were included. Maternal outcomes included those occurring during pregnancy or directly following delivery. Infant outcomes included those measured prenatally until 6 weeks postpartum. Owing to the large number of studies available, only studies published in English were included.

\section{Study population}

Participants were pregnant women who used cannabis during pregnancy but abstained from all other illicit drug use (eg, cocaine, barbiturates), and infants up to 6 weeks after birth who experienced in utero exposure to cannabis.

\section{Outcome measures}

On the basis of the previously published protocol, ${ }^{6}$ studies with the following maternal outcomes were included: pre-eclampsia, eclampsia, postpartum depression, spontaneous delivery, retained placenta, abruption placenta, placenta accrete, placenta previa, postpartum haemorrhage, anaemia, uterine inversion, uterine rupture, vasa previa, oligohydramnios, polyhydramnios, maternal mortality, morning sickness, neonatal nursing, abnormal labour and prenatal care. Fetal outcomes included: ${ }^{6}$ preterm birth (РTB), intrauterine growth restriction, head circumference, infant birth weight, low birth weight (LBW) $(<2500 \mathrm{~g})$, gestational age, fetal length, fetal movement, fetal organ maturity, fetal viability, Apgar score and other neonatal assessments, neonatal intensive care unit or intensive care unit stay (NICU/ICU), days in the hospital, reported neonatal problem such as distress, jaundice, spontaneous abortion, neonatal mortality and resuscitation.

\section{Study selection}

Two reviewers (JKLG and KEC) independently screened titles and abstracts of identified studies to assess their eligibility for inclusion in the review, using an eligibility form based on the study protocol. ${ }^{6}$ Titles and abstracts were first reviewed and duplicates were removed. The reviewers obtained full copies of potentially eligible articles and assessed them independently to determine which articles met the predetermined inclusion criteria. Where there were uncertainties regarding eligibility of studies, all reviewers participated in the inclusion decision (figure 1). 


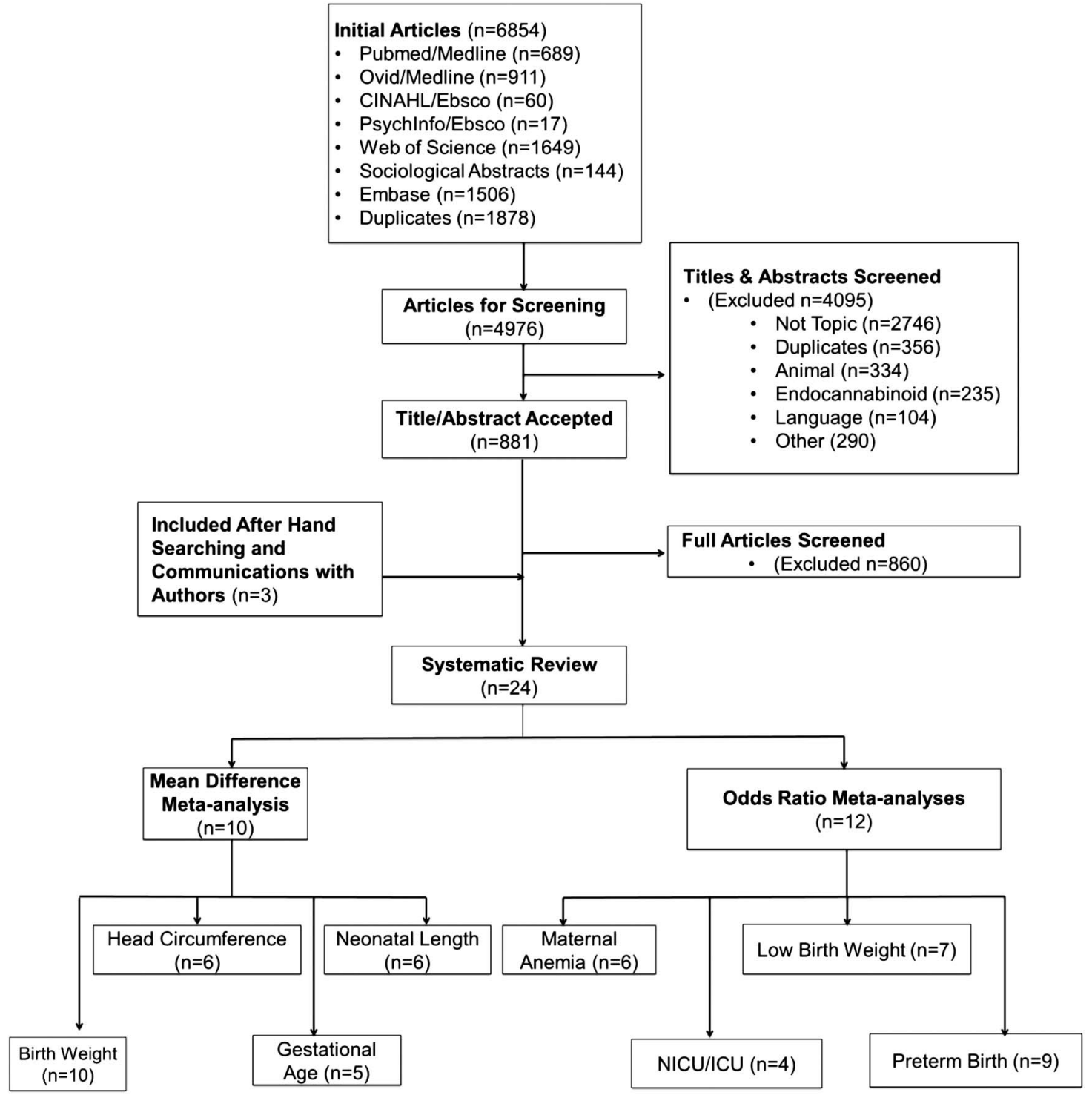

Figure 1 Flow of studies through the review process. ICU, intensive care unit; NICU, neonatal intensive care unit.

\section{Data extraction}

Data from eligible studies were independently extracted by two reviewers (JKLG and SJG), and differences were resolved by discussion and consultation with a third reviewer (JEE). A predesigned data abstraction form was used to extract relevant information. Data extracted from each study included: name of first author, year of publication, study design, location and time of data gathering, participants' demographics, if use of cannabis was self-reported or biologically measured, sample size, variables of interest related to maternal and infant outcomes, study findings, and methods for management of confounding variables. Because studies that focus on use of cannabis during pregnancy often come from a variety of disciplines, OR/risk ratios, mean differences, $p$ values of models, and percentages were extracted.

\section{Bias assessment}

Two authors independently assessed the quality of selected studies using predetermined quality assessment criteria (see online supplement 2). For cross-sectional studies, the National Collaborating Centre for Environmental Health's tool Critical appraisal of crosssectional studies was used to assess the risk of bias. ${ }^{7}$ This tool includes the assessment of study content, method of determining exposure status, comparability of the exposed versus non-exposed group, validation of outcome measures and generalisability. We assigned a composite quality score of 0 (low) to 4 (high). For cohort studies, the Critical Appraisal Skills Programme's Making sense of evidence was used to assess the risk of bias. $^{8}$ This tool includes an assessment of recruitment procedures, measurement of exposure, confounding factors, study results and generalisability. We assigned a composite quality score of 0 (low) to 8 (high). For case-control studies, the Critical Appraisal Skills Programme's Making sense of evidence was used to assess the risk of bias. ${ }^{9}$ This tool uses three broad categories to address study validity, methodology, recruitment styles and appropriateness, validation of exposure 
status and measurements, confounding variables and study outcome. We assigned a composite quality score of 0 (low) to 10 (high). No randomised trials were found.

\section{Assessment of heterogeneity}

Heterogeneity was assessed using the $Q$ test $^{10}$ and the $\mathrm{I}^{2}$ statistic $\left(\mathrm{I}^{2}=100 \% \times(\mathrm{Q}-\mathrm{df}) / \mathrm{Q}\right){ }^{11}$ To assess the $\mathrm{Q}$ test, a $\mathrm{p}$ value of 0.10 or less was considered statistically significant as this value indicates heterogeneity among studies. For studies with a $\mathrm{p}$ value $\leq 0.10, \mathrm{I}^{2}$ was calculated. When using the $\mathrm{I}^{2}$, heterogeneity levels of $\leq 50 \%$ were deemed acceptable. Because of the limited number of articles that met the inclusion criteria for meta-analysis, a subgroup analysis when heterogeneity was more than $50 \%$ could not be conducted.

\section{Data analysis}

Study designs that met inclusion criteria for meta-analysis included: case-control, cross-sectional and cohort. The studies included had participants who used cannabis during pregnancy but did not concurrently use other illicit drugs. For dichotomous variables such as LBW, ORs and $95 \%$ CIs or SEs were either extracted or calculated from the available data. Continuous data, such as birth weight, were extracted as means and SDs. Fixed-effect models were used when heterogeneity was acceptable (ie, $\mathrm{p}>0.10$, or $\mathrm{p} \leq 0.10$ and $\mathrm{I}^{2} \leq 50 \%$ ). If the heterogeneity was not significant $\left(\mathrm{p} \leq 0.10\right.$, but $\left.\mathrm{I}^{2}>50 \%\right)$, the random-effects model was used. The random-effects model accounts for heterogeneity among studies by accounting for differences in the measurement of outcomes among studies. ${ }^{12}$ Pooled results for each maternal or perinatal outcome were calculated using RevMan 5.3. ${ }^{12}$

\section{Maternal and fetal outcomes included in meta-analyses Dichotomous variables}

Only one study explicitly stated that they used WHO's guidelines for classifying anaemia in pregnant women. ${ }^{13}$ WHO defines pregnant women to be anaemic when haemoglobin levels are below $11 \mathrm{~g} / \mathrm{dL} \cdot{ }^{14}$ It is assumed that all the included studies adopted this definition of anaemia. It was also assumed that LBW was defined in the included studies according to WHO's definition: $<2500$ g. $^{15}$ PTB was classified in seven of the eight included studies ${ }^{16-22}$ in accordance with WHO's definition of babies delivered $<37$ weeks gestation. ${ }^{23}$ A neonate's stay in either a (NICU or ICU was grouped together and reported as one variable. ${ }^{16-18} 24$

\section{Continuous variables}

The continuous variables used for this meta-analysis include: birth weight (g), gestational age (weeks), length of neonate $(\mathrm{cm})$ and head circumference $(\mathrm{cm})$. All variables were measured on infant delivery.

\section{RESULTS}

\section{Description of studies}

The initial search results yielded 6854 articles of which 1878 duplicates were found (see figure 1). Of the 4976 unique articles screened, 4095 were excluded based on the abstract or title. In total, 881 full articles were screened, of which $24^{13}$ 16-22 24-39 articles, representing 19 unique studies, ${ }^{13}$ 16-22 24-29 31 36-39 met the inclusion criteria. Ten studies were conducted in the $\mathrm{USA}^{13} 2022 \quad 24252728363839$ five in Canada, ${ }^{31-35}$ three in Australia ${ }^{161821}$ two in The Netherlands ${ }^{29} 30$ and one from each of the following locations: Iran, ${ }^{17}$ Jamaica, ${ }^{37}$ Spain, ${ }^{19}$ and Brazil $^{26}$ (see online supplement 3). The search did not yield any randomised controlled trials. The studies were comprised of 1 cross-sectional, ${ }^{26}$ 1 case-control $^{38}$ and 22 cohort studies. $^{13}$ 16-22 2425 27-29 31-37 $39 \quad 40$ The quality assessment of the studies indicated high study quality (see online supplement 2 ).

\section{Effect of prenatal use of cannabis on maternal health outcomes}

Results on the effects of prenatal use of cannabis on maternal health outcomes are shown in online supplement 3. Anaemia during pregnancy was reported in six studies. 131721222436 One study showed increased odds of being anaemic in mothers who used cannabis during pregnancy; ${ }^{22}$ a null association was demonstrated in five studies. ${ }^{13} 17 \quad 2124 \quad 36$ The fixed-effects model demonstrated statistically significant higher odds of anaemia if the pregnant women smoked cannabis during pregnancy compared with those who did not use cannabis during pregnancy (pooled OR (pOR) 1.36: 95\% CI 1.10 to 1.69) (see online supplement 4, Forest Plot 1). Precipitate labour was discussed in two studies, one demonstrated an increase in odds of having a labour lasting $<3 \mathrm{~h}$ if the mother used cannabis during pregnancy; ${ }^{13}$ the other study demonstrated no association. ${ }^{36}$ Manual removal of the placenta was also discussed in two studies, with one study finding an association ${ }^{36}$ while the other did not. ${ }^{13}$ No association was found between exposure to cannabis in utero and the following variables: maternal diabetes, ${ }^{26}$ rupture of the membranes, ${ }^{172136}$ premature onset of labour, ${ }^{26}$ prolonged labour, ${ }^{13} 36$ dysfunctional labour, ${ }^{36}$ prenatal care, ${ }^{17} 2026$ duration of labour, ${ }^{22}{ }^{36}$ secondary arrest of labour, ${ }^{36}$ elevated blood pressure, ${ }^{21} 2636$ hyperemesis gravidarum, ${ }^{13} 36$ maternal bleeding after 20 weeks, ${ }^{13} 36$ ante/postpartum haemorrhage, ${ }^{1721} 36$ maternal weight gained, ${ }^{13} 22$ maternal postnatal problems, ${ }^{21}$ days in the hospital, ${ }^{21}$ and hormone concentrations. ${ }^{27}$

\section{Effects of prenatal use of cannabis on neonatal outcomes Weight at birth}

As shown in online supplement 3, birth weight was reported as a continuous variable in 10 studies. ${ }^{17} 192122262829313739$ Four of these studies showed a decrease in fetal weight when exposed to cannabis in utero, ${ }^{21} 222829$ while six reported a null 
association. $^{17} \quad 1926 \quad 31 \quad 37 \quad 39$ The random-effects model showed a significant reduction in birth weight of $109.42 \mathrm{~g}$ (95\% CI 38.72 to $\left.180.12, \mathrm{I}^{2}=63 \%\right)$ for infants exposed to cannabis in utero compared with those who were not exposed (see online supplement 4, Forest Plot 2). LBW was reported in seven

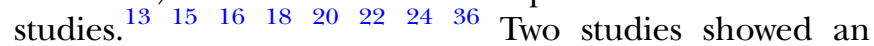
increase in the odds of having an LBW infant when exposed to cannabis in utero; ${ }^{16}{ }^{18}$ five studies reported a null association. ${ }^{13} 20222436$ The random-effects model demonstrated higher odds of LBW in infants' exposure to cannabis in utero compared with those who were not exposed to cannabis in utero (pOR 1.77: 1.04 to 3.01, $\mathrm{I}^{2}=89 \%$ ) (see online supplement 4 , Forest Plot 3 ).

\section{Growth parameters}

Nine studies reported neonatal length at birth; ${ }^{17-21}$ 262835392 reported a significant decrease in gestational length associated with exposure to cannabis in utero, ${ }^{28} 351$ study demonstrated a significant increase, ${ }^{21}$ while the other 6 reported a null association. ${ }^{17-20} 2639$ The random-effects model showed no association between neonatal length and exposure to cannabis in utero ( $\mathrm{pMD}-0.10 ; 95 \%$ CI -0.65 to $0.45, \mathrm{I}^{2}=59 \%$ ) (see supplement 4, Forest Plot 5). There were 10 studies that measured the head circumference of the neonate at birth; ${ }^{17}$ 19-21 2426282935394 studies showed a decrease in head circumference in infants exposed to cannabis in utero, ${ }^{20} \quad 21 \quad 28 \quad 35$ while 6 showed a null association. ${ }^{17} 1924262939$ The random-effects model showed no association between neonatal head circumference and exposure to cannabis in utero ( pMD - 0.31; 95\% CI -0.74 to $0.13, \mathrm{I}^{2}=97 \%$ ) (see online supplement 4 , Forest Plot 6). PTB was reported in nine studies. ${ }^{16-22} 2837$ Three studies showed an increase in odds of PTB ${ }^{16} 1819$ after exposure to cannabis in utero, while six demonstrated a null association. ${ }^{17} \quad{ }^{19-22} 37$ No association was demonstrated between in utero exposure to cannabis and PTB (random effects: pOR=1.29: 0.80 to 2.08, $\mathrm{I}^{2}=85 \%$ ) (see online supplement 4 , Forest Plot 7). Small for gestational age was assessed in two studies; ${ }^{17}$ one study demonstrated an increase in odds of the infant being small for gestational age after exposure to cannabis in utero, ${ }^{18}$ and the other showed a null association. ${ }^{17}$ No association was found between suspected intrauterine growth restriction and exposure to cannabis during pregnancy. $^{13}$

\section{Neonatal assessments}

No association was found between 1 and 5 min Apgar scores of infants exposed to cannabis in

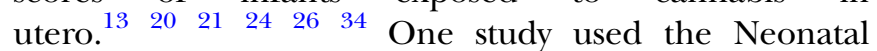
Intensive Care Unit Network Neurobehavioral Scale and found that infants exposed to cannabis in utero experienced few neurobehavioural changes after delivery compared with those infants not exposed to cannabis in utero. $^{26}$ The Brazelton Neonatal Behavioral Assessment Scale (NBAS) was discussed in two studies. ${ }^{33} 37$
Fried $e t a l^{33}$ found that infants exposed to cannabis in utero had decreased visual habituation along with an increased tremor, irritability and startle response. ${ }^{33}$ By contrast, Hayes et $a l^{37}$ found no association between exposure to cannabis in utero and infants' scores on the NBAS until 30 days after birth, at which time those exposed to cannabis had lower automatic stability scores and higher scores on reflexes. ${ }^{37}$ The Prechtl Assessment was discussed in one study. ${ }^{32}$ This study demonstrated an increased association between in utero exposure to cannabis and hand-to-mouth activity, startles and tremor incidence. $^{32}$

\section{Other maternal and child health outcomes}

As shown in online supplement 3, four studies reported associations between in utero exposure to cannabis and the need for placement in NICU/ICU; ${ }^{16-18} 24$ three studies $^{16-18}$ showed a positive association of infants being placed in the NICU/ICU and exposure to cannabis in utero, and one study demonstrated a null association. $^{24}$ The random-effects model demonstrated higher odds of an NICU/ICU stay if the infant was exposed to cannabis in utero compared with those who were not exposed to cannabis in utero (pOR 2.02, 1.27 to 3.21, $\mathrm{I}^{2}=78 \%$ ) (see online supplement 4 , Forest Plot 8 ). Five

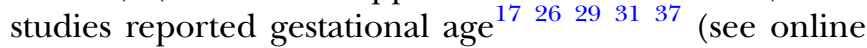
supplement 3). Four of these studies showed a null association between gestational age and exposure to cannabis in utero; ${ }^{17} \quad 26 \quad 29 \quad 37$ one study demonstrated a significant decrease in gestational age after exposure to cannabis in utero. ${ }^{31}$ The fixed-effects model showed no association between in utero exposure to cannabis and gestational age (pooled mean difference ( $\mathrm{pMD}):-0.20$; $95 \%$ CI -0.62 to 0.22 ) (see online supplement 4 , Forest Plot 4). No association was found between exposure to cannabis in utero and the following variables: days the infant stayed in the hospital; ${ }^{17} 2126$ jaundice ${ }^{26} 36$ resuscitation; ${ }^{13} 36$ respiratory distress syndrome; ${ }^{132426}$ perinatal

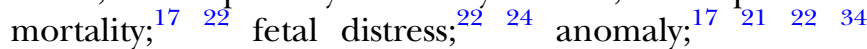
abnormal fetal tests; ${ }^{13}$ blood transfusion; ${ }^{24}$ abruptio; ${ }^{22}$ spontaneous abortion; ${ }^{34} 38$ needing intubation after delivery; ${ }^{24}$ hypoglycaemia ${ }^{26}$ and sepsis. ${ }^{24}$ Ammenheuser et $a l^{25}$ found an increase in mutant lymphocytes in the mother and the fetus after women used cannabis while pregnant. No association was seen in women who use cannabis during pregnancy and chromosomal abnormalities. ${ }^{38}$ Fetuses of mothers who used cannabis throughout pregnancy had a significantly smaller diameter of their inner aorta and their pulmonary peak systolic velocity was lower than fetuses of mothers who did not use cannabis during pregnancy. ${ }^{30}$ The pulse index and resistance index of placental blood flow was significantly higher for those fetuses exposed to cannabis in utero. ${ }^{30}$ No association was seen in cardiac blood flow and the following variables: fetal heart rate of the aorta or pulmonary arteries, the inner diameter of the pulmonary artery, and aortic peak systolic velocity. ${ }^{30}$ 


\section{DISCUSSION}

Medical and social use of cannabis is rapidly becoming more acceptable in the USA and around the world. ${ }^{2} 41$ The effects of in utero exposure to other illicit drugs, such as cocaine, have been widely studied. Less is known about the effects of cannabis on fetal growth and development, or its effects on pregnant women. This systematic review summarised and critically appraised the existing literature on the effects of prenatal exposure to cannabis on women and their neonates. This meta-analysis demonstrated that women who used cannabis during pregnancy had an increase in the odds of anaemia compared with women who did not use cannabis during pregnancy. Infants exposed to cannabis in utero had a decrease in birth weight compared with infants whose mothers did not use cannabis during pregnancy. Infants exposed to cannabis in utero were also more likely to need placement in the NICU/ICU compared with infants whose mothers avoided using cannabis during pregnancy.

Overall, very few studies reported overlapping maternal outcomes. Anaemia was the most widely discussed maternal outcome in the cannabis-pregnancy literature. This was not surprising, as anaemia is part of the screening panels during prenatal care in most countries. Only one of six showed an increase in the odds of having anaemia in mothers who used cannabis during pregnancy compared with those who did not use cannabis during pregnancy. However, with a sample size of 8350 , this study had the largest sample size of all the studies in this review, and accounted for the greatest weight in the meta-analysis. ${ }^{22}$ More research is needed to determine whether there is any association between maternal anaemia and use of cannabis during pregnancy. Therefore, it is recommended that these study results be interpreted with caution until future studies are completed. Results from this systematic review also indicated that very few studies showed any association, negative or positive, between use of cannabis during pregnancy and adverse maternal outcomes. Because few studies reported overlapping maternal outcomes, the effects of exposure to cannabis on maternal outcomes will remain unclear until more studies are completed.

This meta-analysis demonstrated that the outcome of an infant exposed to cannabis in utero results in low birth weight when measured both as a dichotomous and continuous variable. LBW is associated with an increase in morbidity and mortality in infants. ${ }^{42}{ }^{43}$ Long-lasting negative consequences of LBW include: neurosensory impairments; ${ }^{44}{ }^{45}$ decrease in height; ${ }^{44}{ }^{45}$ decreased IQ and educational achievement; ${ }^{44} 45$ and increased psychopathology. ${ }^{46}$ Educating women and physicians on the possible risks associated between lower birth weight and in utero exposure to cannabis is warranted.

Infants exposed to cannabis in utero had an increased placement in the NICU/ICU. Costs associated with the NICU are among the highest in healthcare, in and outside the USA. ${ }^{47-50}$ As this was demonstrated in three ${ }^{16-18}$ of four ${ }^{24}$ studies, it warrants further attention by doctors and policymakers alike. The effects of in utero exposure to cannabis on fetal growth parameters and other fetal outcomes-that is, gestational age, length, head circumference-were not demonstrated in the meta-analysis. However, because the results of all growth parameters remained fairly inconsistent in the literature, more studies are needed to determine the association between use of cannabis and fetal growth parameters.

The effects of use of cannabis on neonatal assessments were rarely discussed in the literature. Apgar score was the most discussed neonatal assessment; no significant associations were demonstrated between in utero exposure to cannabis and Apgar scores. Results of other neonatal assessments, such as the NBAS or Prechtl, were rarely discussed in the literature and warrant further attention.

This systematic review and meta-analysis are not without limitations. There remains a gap in the literature assessing the effects of cannabis excluding other illicit drug use. As use of cannabis gains social and medical acceptance, understanding its effects on maternal and fetal outcomes remains essential. As many cannabis users are often tobacco or alcohol users, determining a cannabis-only effect (excluding the presence of tobacco and alcohol) was currently not possible in this systematic review and meta-analysis with the available literature. ${ }^{51}$ It is well established that both tobacco and heavy alcohol use increase adverse fetal outcomes such as LBW, PTB and being small for gestational age, ${ }^{52} 53$ therefore, it is unknown if the effects found in this manuscript are related to cannabis or are a by-product of alcohol and tobacco use. Controlling for potential confounding variables is essential to understanding any relationship. Because studying the relationship between cannabis and maternal and fetal outcomes is fairly recent, future research needs to take confounding and utilisation of appropriate control groups into consideration.

Also, numerous articles reported unique maternal and fetal outcomes not reported in other studies; therefore, definitive conclusions could not be drawn. Moreover, very few outcomes were measured in standardised methods. Maternal anaemia, PTB and LBW all have standard cut-offs that are used for clinical practice. Although these outcomes were all frequently reported in the literature, few studies defined them in their manuscripts. Furthermore, reliance on self-reported measures of use of cannabis, may have underestimated the prevalence of drug use during pregnancy due to social desirability. More studies that assess drug use using biological screening panels along with self-report measures are needed to estimate self-report bias. Lastly, studies often failed to report maternal age, or only have a mean and SD. Therefore, the authors of the current manuscript were not able to restrict this systematic review and meta-analysis by maternal age. There was an 
initial attempt to restrict our inclusion criteria to women 18 years or older; however, the search results did not yield enough studies to warrant this age restriction.

By conclusion, the effects of cannabis on maternal and fetal outcomes remain generally unknown. Results from this systematic review and meta-analysis demonstrated that future research in the area of cannabis and maternal and fetal health needs to employ stricter inclusion criteria and exclude polyillicit substance use. There does appear to be negative consequences associated with in utero exposure to cannabis, including decrease in birth weight and a need for placement in the NICU/ ICU. However, more research is needed to further assess these relationships in more homogeneous populations. Pregnant women could benefit from health education on the potential adverse effects of use of cannabis during pregnancy. As use of cannabis continues to become socially acceptable in many countries, understanding the effects on maternal and fetal health should become a global priority.

\section{Author affiliations}

${ }^{1}$ Department of Epidemiology and Biostatistics, Mel and Enid Zuckerman College of Public Health, University of Arizona, Tucson, Arizona, USA ${ }^{2} \mathrm{Mel}$ and Enid Zuckerman College of Public Health, University of Arizona, Phoenix, Arizona, USA

${ }^{3}$ Department of Obstetrics and Gynecology, University of Arizona, Tucson, Arizona, USA

${ }^{4}$ Arizona Health Sciences Library, Mel and Enid Zuckerman College of Public Health, University of Arizona, Tucson, Arizona, USA

${ }^{5}$ Department of Health Promotion Sciences, Mel and Enid Zuckerman College of Public Health, University of Arizona, Tucson, Arizona, USA

${ }^{6}$ Arizona Department of Health Services, Phoenix, Arizona, USA

Contributors CBR and JEE conceived the idea for the study and oversaw all aspects of protocol development and final review. JKLG and AN made substantial contributions to the conception and design of the project. SJG and KEC assisted in the logistics of data collection. CC edited and commented on drafts of the manuscript. All authors were involved in manuscript preparation.

Funding Financial support for this work was provided by the Arizona Department of Health Services, Phoenix, Arizona, USA.

\section{Competing interests None declared.}

Provenance and peer review Not commissioned; externally peer reviewed.

Data sharing statement No additional data are available.

Open Access This is an Open Access article distributed in accordance with the Creative Commons Attribution Non Commercial (CC BY-NC 4.0) license, which permits others to distribute, remix, adapt, build upon this work noncommercially, and license their derivative works on different terms, provided the original work is properly cited and the use is non-commercial. See: http:// creativecommons.org/licenses/by-nc/4.0/

\section{REFERENCES}

1. WHO. Management of substance abuse: Cannabis. 7 July 2015 http://www.who.int/substance_abuse/facts/cannabis/en/

2. Crime, U.N.O.o.D.a. World drug report. 2014. http://www.unodc.org/ documents/wdr2014/World_Drug_Report_2014_web.pdf

3. Degenhardt L, Whiteford H, Hall WD. The Global Burden of Disease projects: what have we learned about illicit drug use and dependence and their contribution to the global burden of disease? Drug Alcohol Rev 2014;33:4-12.

4. Board, I.N.C, U.N.V.I. Centre, and Austria. International Narcotics Control Board: 2013 Annual Report, 2013.
5. English D, Hulse GK, Milne E, et al. Maternal cannabis use and birth weight: a meta-analysis. Addiction 1997;92:1553-60.

6. Gunn JK, Rosales CB, Center KE, et al. The effects of prenatal cannabis exposure on fetal development and pregnancy outcomes: a protocol. BMJ open 2015;5:e007227.

7. Health, N.C.C.f.E. and (NCCEH). A Primer for Evaluating the Quality of Studies onEnvironmental Health Critical Appraisal of Cross-Sectional Studies. 2011 (updated 5 June 2014). http://www. ncceh.ca/sites/default/files/Critical_Appraisal_Cross-Sectional_ Studies Aug 2011.pdf

8. UK, C.A.S.P.C. Making sense of evidence: 12 questions to help you make sense of a cohort study. 2013 (cited Nov 2014). http://www. casp-uk.net/\#!casp-toolschecklists/c18f8

9. UK, C.A.S.P.C. Making sense of evidence: 11 questions to help you make sense of a case control study. 2013. http://www.casp-uk.net/\#! casp-toolschecklists/c18f8

10. Cochran WG. The combination of estimates from different experiments. Biometrics 1954;10:101-29.

11. Higgins JP, Thompson SG. Quantifying heterogeneity in a meta-analysis. Stat Med 2002;21:1539-58.

12. Review Manager (RevMan). 2014, Copenhagen: The Nordic Cochrane Centre, The Cochrane Collaboration.

13. Greenland S, Staisch KJ, Brown N, et al. Effects of marijuana on human pregnancy, labor, and delivery. Neurobehav Toxicol Teratol 1982;4:447-50.

14. WHO. Development of indicators for monitoring progress towards health for all by the year 2000. 1981 (cited Sept 2015) http://apps. who.int/iris/handle/10665/40672

15. Wardlaw TM. Low birthweight: country, regional and global estimates. UNICEF, 2004.

16. Bonello MR, Xu F, Li Z, et al. Mental and behavioral disorders due to substance abuse and perinatal outcomes: a study based on linked population data in New South Wales, Australia. Int $J$ Environ Res Public Health 2014;11:4991-5005.

17. Gargari SS, Fallahian M, Haghighi L, et al. Maternal and neonatal complications of substance abuse in Iranian pregnant women. Acta Med Iran 2012;50:411-16.

18. Hayatbakhsh MR, Flenady VJ, Gibbons KS, et al. Birth outcomes associated with cannabis use before and during pregnancy. Pediatr Res 2012;71:215-19.

19. Lozano J, García-A-Algar O, Marchei E, et al. Prevalence of gestational exposure to cannabis in a Mediterranean city by meconium analysis. Acta Paediatr 2007;96:1734-7.

20. Ostrea EM, Ostrea AR, Simpson PM. Mortality within the first 2 years in infants exposed to cocaine, opiate, or cannabinoid during gestation. Pediatrics 1997;100:79-83.

21. Quinlivan JA, Evans SF. The impact of continuing illegal drug use on teenage pregnancy outcomes-a prospective cohort study. BJOG 2002;109:1148-53.

22. Witter FR. Niebyl JR. Marijuana use in pregnancy and pregnancy outcome. Am J Perinatol 1990;7:36-8.

23. [No authors listed]. WHO: recommended definitions, terminology and format for statistical tables related to the perinatal period and use of a new certificate for cause of perinatal deaths. Modifications recommended by FIGO as amended October 14, 1976. Acta Obstet Gynecol Scand 1977;56:247-53.

24. Berenson AB, Wilkinson GS, Lopez LA. Effects of prenatal care on neonates born to drug-using women. Subst Use Misuse 1996;31:1063-76.

25. Ammenheuser MM, Berenson AB, Babiak AE, et al. Frequencies of hprt mutant lymphocytes in marijuana-smoking mothers and their newborns. Mut Res 1998;403:55-64.

26. de Moraes Barros MC, Guinsburg R, De Araújo Peres C, et al. Exposure to marijuana during pregnancy alters neurobehavior in the early neonatal period. J Pediatr 2006; 149:781-7.

27. Braunstein GD, Buster JE, Soares JR, et al. Pregnancy hormone concentrations in marijuana users. Life Sci 1983;33:195-9.

28. Chasnoff IJ, Griffith DR, Freier C, et al. Cocaine/polydrug use in pregnancy: two-year follow-up. Pediatrics 1992;89:284-9.

29. El Marroun $\mathrm{H}$, Tiemeier $\mathrm{H}$, Steegers EA, et al. Intrauterine cannabis exposure affects fetal growth trajectories: the Generation R Study. J Am Acad Child Adolesc Psychiatry 2009;48:1173-81.

30. El Marroun $\mathrm{H}$, Tiemeier $\mathrm{H}$, Steegers EA, et al. A prospective study on intrauterine cannabis exposure and fetal blood flow. Early Hum Dev 2010;86:231-6.

31. Fried $P$, Watkinson $B$, Willan A. Marijuana use during pregnancy and decreased length of gestation. Am J Obstet Gynecol 1984;150:23-7.

32. Fried PA, Watkinson B, Dillon RF, et al. Neonatal neurological status in a low-risk population after prenatal exposure to cigarettes, marijuana, and alcohol. J Dev Behav Pediatr 1987;8:318-26. 
33. Fried PA, Makin J. Neonatal behavioural correlates of prenatal exposure to marihuana, cigarettes and alcohol in a low risk population. Neurotoxicol Teratol 1987;9:1-7.

34. Fried PA. Marijuana use during pregnancy: consequences for the offspring. Semin Perinatol 1991;15:280-7.

35. Fried PA, Watkinson B, Gray R. Growth from birth to early adolescence in offspring prenatally exposed to cigarettes and marijuana. Neurotoxicol Teratol 1999;21:513-25.

36. Greenland S, Richwald GA, Honda GD. The effects of marijuana use during pregnancy. II. A study in a low-risk home-delivery population. Drug Alcohol Depend 1983;11:359-66.

37. Hayes JS, Lampart R, Dreher MC, et al. Five-year follow-up of rural Jamaican children whose mothers used marijuana during pregnancy. West Indian Med J 1991;40:120-3.

38. Kline J, Hutzler M, Levin B, et al. Marijuana and spontaneous abortion of known karyotype. Paediatr Perinat Epidemiol 1991;5:320-32

39. Shankaran S, Das A, Bauer CR, et al. Association between patterns of maternal substance use and infant birth weight, length, and head circumference. Pediatrics 2004;114:e226-34

40. Fried $\mathrm{P}$, Buckingham $\mathrm{M}$, Von Kulmiz $\mathrm{P}$. Marijuana use during pregnancy and perinatal risk factors. Am J Obstet Gynecol 1983;146:992-4.

41. Nosyk B, Wood E, Kerr T. The rise of marijuana and the fall of cocaine in the United States: for better, for worse? Addiction 2015;110:737-8.

42. McCormick MC. The contribution of low birth weight to infant mortality and childhood morbidity. N Engl J Med 1985;312:82-90.

43. Horbar JD, Carpenter JH, Badger GJ, et al. Mortality and neonatal morbidity among infants 501 to 1500 grams from 2000 to 2009 . Pediatrics 2012:129:1019-26.
44. Hack M, Flannery DJ, Schluchter M, et al. Outcomes in young adulthood for very-low-birth-weight infants. $N$ Engl J Med 2002;346:149-57

45. Black SE, Devereux PJ, Salvanes K. From the cradle to the labor market? The effect of birth weight on adult outcomes. National Bureau of Economic Research, 2005.

46. Hack M, Youngstrom EA, Cartar L, et al. Behavioral outcomes and evidence of psychopathology among very low birth weight infants at age 20 years. Pediatrics 2004;114:932-40.

47. Cömert S, Ağzıkuru T, Akin Y, et al. The cost analysis of preterm infants from a NICU of a state hospital in Istanbul. Iran J Pediatr 2012;22:185.

48. Cheah IG, Soosai AP, Wong SL, et al. Cost-effectiveness analysis of Malaysian neonatal intensive care units. J Perinatol 2005;25:47-53.

49. Profit J, Lee D, Zupancic JA, et al. Clinical benefits, costs, and cost-effectiveness of neonatal intensive care in Mexico. PLoS Med 2010;7:e1000379.

50. Prinja S, Manchanda N, Mohan P, et al. Cost of neonatal intensive care delivered through district level public hospitals in India. Indian Pediatr 2013;50:839-46.

51. Jaques SC, Kingsbury A, Henshcke $P$, et al. Cannabis, the pregnant woman and her child: weeding out the myths. J Perinatol 2014;34:417-24.

52. Patra J, Bakker R, Irving H, et al. Dose-response relationship between alcohol consumption before and during pregnancy and the risks of low birthweight, preterm birth and small for gestational age (SGA)-a systematic review and meta-analyses. BJOG 2011;118:1411-21.

53. Janisse JJ, Bailey BA, Ager J, et al. Alcohol, tobacco, cocaine, and marijuana use: relative contributions to preterm delivery and fetal growth restriction. Subst Abus 2014;35:60-7. 